\title{
Cokrigagem O rdinária versus Krigagem com Deriva Externa: Aplicações para a Avaliação do Nível Potenciométrico em um Aquífero Livre
}

\author{
O rdinary Co-kriging versus Kriging with External Drift Correction: \\ Applications to the Evaluation of the Potentiometric Level of a Free Aquifer
}

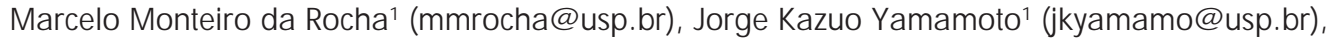 \\ Helano Regis da Nóbrega Fonteles² (helano.fonteles@dnpm.gov.br) \\ ${ }^{1}$ Departamento de Geologia Sedimentar e Ambiental - Instituto de G eociências - USP \\ R. do Lago 562, CEP 05508-080, São Paulo, SP, BR \\ 2Departamento Nacional de Produção Mineral - DNPM - $21^{\circ}$ Distrito, Teresina, PI, BR
}

Recebido em 06 de junho de 2008; aceito em 15 de setembro de 2008

\begin{abstract}
RESUMO
Mapas de curvas equipotenciais são obtidos a partir de poços de monitoramento espalhados pela região que se deseja representar. Estes mapas são elaborados a partir dos mais variados métodos de interpolação, porém nem sempre a densidade de informações de cota potenciométrica disponível é suficiente para a realização de uma boa estimativa. Nestes casos pode-se lançar mão da utilização de interpoladores mais complexos, como por exemplo, a cokrigagem ou a krigagem com deriva externa, que são métodos que utilizam mais de uma fonte de informações, complementares, para a interpolação de uma dada característica. Neste trabalho foram aplicadas a cokrigagem ordinária, a krigagem com deriva externa e a krigagem com deriva externa com correção do efeito de suavização para a elaboração do mapa potenciométrico de um aquífero livre na porção NE da região metropolitana de Fortaleza, estado do Ceará. A cokrigagem ordinária, dos três métodos, é sem dúvida o mais trabalhoso e o que apresentou os piores resultados uma vez que a correlação entre a cota potenciométrica e a cota altimétrica diminuiu após as estimativas. A krigagem com deriva externa apresentou-se como uma técnica viável e mais simples de ser aplicada que a cokrigagem ordinária além de ter apresentado melhores resultados uma vez que a correlação entre as duas informações não foi alterada significativamente.
\end{abstract}

Palavras-chave: geoestatística multivariada, cokrigagem, krigagem com deriva externa, efeito de suavização, mapa potenciométrico, hidrogeologia.

\begin{abstract}
Equipotential maps are obtained from monitoring wells distributed over the study area. These maps can be built using several interpolation methods, nevertheless the available potentiometric information is not sufficient to ensure good estimation for unsampled locations. In these cases one can use more complex interpolators such as normal cokriging and external drift kriging, with and without correction of the smoothing effect. These methods consider not only the variable to be estimated but also another correlated variable. Thus, we can use information coming from a secondary source to estimate the main variable. For instance, elevation is well correlated with potentiometric data and it is very easy to acquire over the study area. In this paper cokriging, kriging with external drift, and kriging with external drift after correcting the smoothing effect have been used for the preparation of the potentiometric map of a free aquifer in the NE part of Fortaleza City, Ceará State. Among the three tested methods cokriging gave the worst results because of the instability of the system of cokriging equations. Kriging with an external drift seems to be appropriate since it is simpler to implement than cokriging, and it depends on correlation of the secondary information with the variable to be estimated. Furthermore, kriging with external drift after correcting the smoothing effect did not improve the results because available information is not uniformly distributed over the study area. Actually, errors coming from portions with few data are scattered out over other better sampled portions resulting in poorer estimates.
\end{abstract}

Keywords: multivariate geostatistics, cokriging, external drift kriging, smoothing effect, potenciometric map, hydrogeology. 


\section{INTRODUÇÃO}

Medidas de cota potenciométrica são tomadas em poços de monitoramento, os quais envolvem custos na obtenção das mesmas. Estas informações são, então, utilizadas na elaboração de mapas de curvas equipotenciais. Tradicionalmente, estas curvas são obtidas pela interpretação manual das informações coletadas nos poços de monitoramento adicionadas a dados oriundos da cota altimétrica de lagos, rios e, porventura, do mar. Havendo a existência de quantidade suficiente de poços de monitoramento e ainda das cotas altimétricas, podem ser geradas curvas equipotenciais por meio de interpolação matemática, como exemplificado em Sturaro e Landim (1994). Esta automatização do processo pode gerar, quando na ausência de boa distribuição espacial dos poços e da altimetria, artefatos denominados "olhos de boi” (concentração anômala de curvas de isovalores no entorno de um único ponto ou pequeno número de pontos), que são feições indesejadas porque não possuem significado. Para contornar esta situação, podem-se adotar procedimentos distintos, o primeiro é interpretar o mapa de curvas gerado matematicamente e traçar novas curvas manualmente corrigindo os artefatos. Pode-se também resolver este problema pela geração artificial de dados, utilizando, por exemplo, a média aritmética entre dois pontos amostrais, nas áreas onde existe menor densidade de informação, minimizando assim a geração de tais feições. Os métodos matemáticos utilizados são o Inverso da Potência da Distância (IPD) e a Krigagem Ordinária, que são dependentes de grande quantidade de dados com boa distribuição espacial e, mesmo assim, podem ter artefatos em seus resultados. Um exemplo dessa situação pode ser visto em Yamamoto (1998), na qual a utilização de potências elevadas na interpolação pelo inverso da distância gera áreas planas (artefatos) em torno dos pontos de dados.

Quando ocorre menor quantidade de informações e várias fontes correlacionáveis, por exemplo, dados de alguns poços de monitoramento e de elevação do terreno, pode-se utilizar a cokrigagem em substituição à krigagem ordinária. Este procedimento, embora viável, não apresenta ganho significativo em relação à krigagem ordinária, quando a malha amostral da cota potenciométrica é suficiente para reproduzir o fenômeno espacial subjacente, conforme demonstrado em Fonteles et al. (2006). O pequeno ganho sobressai quando os resultados são analisados sob a ótica do trabalho despendido e conhecimento necessário para a aplicação do método.

Outra forma de abordar o problema é pelo método de krigagem com deriva externa, que modela a variável primária, neste caso a cota potenciométrica, com base na forma da topografia e para tal utiliza o variograma do resíduo gerado entre a variável primária e a variável secundária. Este método requer apenas que os dados da variável secundária sejam multicolocalizados, ou seja, que a cota topográfica esteja presente em todos os pontos da malha regular a ser estimada e ainda em todos os pontos onde a cota potenciométrica tenha sido amostrada.

Uma característica comum a todos os métodos de estimativa que utilizam o conceito de médias móveis é o efeito de suavização. Este efeito faz com que valores extremos de uma distribuição não sejam reproduzidos, ou seja, os valores baixos da distribuição serão superestimados e os valores altos subestimados. A depender do que se deseja representar, esta característica é um revés tornandose um problema a ser evitado. Neste sentido, vários autores como Olea e Pawlowsky (1996); Journel, Kyriakids e Mao (2000) e Yamamoto (2005) propuseram diversos algoritmos para corrigir tal efeito. Neste estudo, será utilizado o algoritmo de Yamamoto (2005) aplicado à krigagem com deriva externa. A correção de Yamamoto (2005) não será aplicada à cokrigagem, pois ela é baseada na validação cruzada, como o método proposto por Olea e Pawlowsky (1996) e também no desvio de interpolação proposto por Yamamoto (2000). Como o desvio de interpolação não é uma medida de incerteza válida ou mensurável, na cokrigagem, devido à condição de que todos os pesos de estimativa sejam positivos, o que não ocorre na coestimativa, não é possível corrigir o efeito de suavização.

Assim sendo, este trabalho tem como objetivos a comparação entre a cokrigagem ordinária e a krigagem com deriva externa com e sem correção do efeito de suavização. Estes três métodos serão aplicados aos dados de cota potenciométrica de um aquífero livre na região metropolitana de Fortaleza, auxiliados pela informação da cota altimétrica da região do aquífero.

\section{MÉTODOS}

\section{Krigagem ordinária}

O mais usual dos métodos, a krigagem ordinária, é um melhor estimador linear não enviesado, com mínima variância de erro, no sentido dos mínimos quadrados.

Para a realização da krigagem assumem-se a estacionariedade dos dados assim como sua ergodicidade, tanto um quanto outro resultam em variogramas experimentais bem estruturados e com patamar definido. Ajusta-se ao variograma experimental o modelo teórico de variograma, que nada mais é do que uma função matemática predefinida.

O modelo teórico de variograma permite o acesso aos valores de variância espacial para quaisquer distâncias e em qualquer direção dentro do domínio. Estes valores, utilizados no sistema de equações de krigagem, permitirão 
derivar os pesos da krigagem, os quais serão empregados no estimador da krigagem conforme, respectivamente:

$$
\left\{\begin{array}{l}
\sum_{i=1}^{n} \lambda_{i} \gamma\left(x_{i}-x_{n}\right)+\mu=\gamma\left(x_{0}-x_{i}\right) i=1,2, \ldots, n \\
\sum_{i=1}^{n} \lambda_{i}=1
\end{array}\right.
$$

e

$$
Z^{*}\left(X_{0}\right)=\sum_{i=1}^{n} \lambda_{i} z\left(x_{i}\right)
$$

onde $\lambda_{\mathrm{i}}$ é o ponderador da krigagem associada ao i-ésimo ponto amostral; $\gamma\left(x_{i}-x_{n}\right)$ é a variância espacial entre os pontos amostrais de uma dada vizinhança; $\gamma\left(x_{i}-x_{0}\right)$ é a variância espacial entre o ponto a ser estimado e o i-ésimo ponto amostral; $Z^{*}\left(X_{0}\right)$ é o ponto $x_{0}$ estimado por krigagem ordinária e $z\left(x_{i}\right)$ é o valor do i-ésimo ponto amostral na vizinhança de $Z^{*}\left(X_{0}\right)$.

\section{Cokrigagem ordinária}

Os métodos de cokrigagem definem-se como estimativas multivariadas por utilizarem várias fontes de informação de maneira complementar. Do mesmo modo que na krigagem ordinária, para o cálculo da cokrigagem deve-se assumir a estacionariedade de segunda ordem e a ergodicidade dos variogramas. Deve-se também verificar a presença do modelo de corregionalização linear que se traduz em variogramas diretos proporcionais entre si, variogramas cruzados bem estruturados e também proporcionais aos variogramas diretos. A proporcionalidade entre variâncias pode, segundo Isaacs e Srivastava (1989), ser validada mostrando-se que a condição de positividade definida é atendida em cada estrutura ajustada, conforme:

$$
\left|\begin{array}{ll}
\gamma_{1,1} & \gamma_{1,2} \\
\gamma_{2,1} & \gamma_{2,2}
\end{array}\right| \geq 0
$$

ou simplesmente verificando que:

$$
\gamma_{1,1} * \gamma_{2,2} \geq \gamma_{1,2}^{2}
$$

onde $\gamma_{1,1}$ é a variância espacial direta da variável primária; $\gamma_{2,2}$ é a variância espacial direta da variável secundária e $\gamma_{1,2}$ é a variância espacial cruzada da variável primária pela variável secundária.

O variograma cruzado é uma extensão natural do variograma direto e é calculado como: $\gamma_{1,2}(h)=\frac{1}{2 n} \sum_{i=1}^{n}\left\{\left[z^{1}\left(x_{i}\right)-z^{1}\left(x_{i}+h\right)\right] *\left[z^{2}\left(x_{i}\right)-z^{2}\left(x_{i}+h\right)\right]\right\}$

onde $\gamma_{1,2}(h)$ é o variograma cruzado entre a variável primária e a secundária; $z^{1}\left(x_{i}\right)$ é o valor da variável primária no ponto $x_{i} ; z^{1}\left(x_{i}+h\right)$ é o valor da variável primária no ponto $x_{i}$ adicionado de uma distância $h ; z^{2}\left(x_{i}\right)$ é o valor da variável secundária no ponto $x_{i}$; $z^{2}\left(x_{i}+h\right)$ é o valor da variável secundária no ponto $x_{i}$ adicionado de uma distância $h$ e $n$ é o número de pares de pontos formados para uma dada distância $h$.

O estimador da cokrigagem ordinária pode ser escrito como:

$$
Z_{C K}^{*}=\sum_{k}^{N} \sum_{i=1}^{n} \lambda_{i}^{k} z^{k}\left(x_{i}\right)
$$

onde $Z_{C K}^{*}$ é o estimador da cokrigagem no ponto $x_{0}$; $N$ é o número de variáveis utilizadas; $\lambda_{i}^{k}$ é o i-ésimo ponderador da k-ésima variável e $Z^{k}\left(x_{i}\right)$ é o valor da k-ésima variável no i-ésimo ponto amostral. Deve-se destacar que o sobrescrito $k$ não indica potenciação, mas sim apenas o contador que indica a variável (primária ou secundária).

O sistema de cokrigagem ordinária pode ser escrito como (Wackernagel, 1998):

$$
\begin{aligned}
& \left\{\begin{array}{l}
\sum_{k=1}^{\mathrm{N}} \sum_{i=1}^{n} \lambda_{i}^{k} \gamma_{k l}\left(x_{i}-x_{j}\right)+\mu_{k}=\gamma_{k k_{0}}\left(x_{i}-x_{0}\right) \\
\sum_{i=1}^{n} \lambda_{i}^{k}=\delta_{k k_{0}}
\end{array}\right. \\
& \text { com } \quad \delta_{k k_{0}}=\left\{\begin{array}{l}
1 \text { se } k=k_{0} \\
0 \text { se } k \neq k_{0}
\end{array}\right.
\end{aligned}
$$

A condição de restrição $\delta{ }_{k k_{0}}$ faz com que sempre haja pesos negativos para que o somatório de $\lambda$ seja zero. Deste modo, a correção do efeito de suavização, conforme proposta por Yamamoto (2005) não pode ser aplicada.

\section{Krigagem com deriva externa}

A krigagem com deriva externa é um método multivariado que permite a estimativa da variável primária considerando-se as informações de uma variável secundária extensivamente amostrada e, conforme já mencionado, os dados da variável secundária precisam ser multicolocalizados.

Neste método de estimativa, não existe a necessidade da variável primária ser estacionária, pois a variância espa- 
cial utilizada provém dos resíduos entre a variável primária e a variável secundária, que são estacionárias e ergódicas. Porém, a variável primária e a secundária e o modelo de deriva externa devem obrigatoriamente apresentar alto grau de dependência linear.

Considere-se $Z^{*}(x)$ como a variável primária e $\mathrm{Y}(x)$ a variável secundária, ambas apresentando dependência linear, conforme escreve Wackernagel (1998):

$$
\mathrm{E}[\mathrm{Z}(x)]=a_{0}+b_{1} y(x) .
$$

Ainda segundo Wackernagel (1998), o estimador da krigagem com deriva externa, $z_{K E D}^{*}\left(x_{o}\right)$, pode ser escrito como:

$$
z_{K E D}^{*}\left(x_{0}\right)=\sum_{i=1}^{n} \lambda_{i} z\left(x_{i}\right)
$$

com a primeira condição de não enviesamento sendo:

$$
\sum_{i=1}^{n} \lambda_{i}=1
$$

e a segunda condição de não enviesamento como:

$$
y\left(x_{0}\right)=\sum_{i=1}^{n} \lambda_{i} y\left(x_{i}\right) .
$$

Os valores dos ponderadores são obtidos pela resolução de um sistema de equações lineares, conforme:

$$
\left\{\begin{array}{l}
\sum_{i=1}^{n} \lambda_{i} C_{0}^{r}\left(x_{j}-x_{i}\right)-\mu_{1}-\mu_{2} y\left(x_{i}\right)=C^{r}\left(\begin{array}{l}
\left(x_{i}-x_{0}\right) \\
\text { para } i=1, \ldots, n
\end{array}\right. \\
\sum_{i=1}^{n} \lambda_{i}=1 \\
\sum_{i=1}^{n} \lambda_{i} y\left(x_{i}\right)=y\left(x_{0}\right)
\end{array}\right.
$$

onde $\mu_{1}$ e $\mu_{2}$ são os multiplicadores de Lagrange e $C^{r}\left(x_{j}-x_{i}\right)$ é a covariância centrada dos resíduos, entre a variável primária e a secundária, da j-ésima amostra em relação à i-ésima.

\section{Correção do efeito de suavização na krigagem com deriva externa}

Como mencionado anteriormente, a correção do efeito de suavização proposta por Yamamoto (2005) utilizase da validação cruzada e do desvio de interpolação de
Yamamoto (2000). Este método de correção foi definido originalmente para ser aplicado à krigagem ordinária, porém como o estimador da krigagem com deriva externa é baseado em médias ponderadas, é possível derivar a variância de interpolação e assim adaptar o método de correção à krigagem com deriva externa.

A variância de interpolação é calculada, segundo Yamamoto (2000), como:

$$
s_{0}^{2}=\sum \lambda_{i}\left[z\left(x_{i}\right)-z_{K O}^{*}\left(x_{0}\right)\right]^{2}
$$

que por analogia pode ser escrito como:

$$
s_{0}^{2}=\sum \lambda_{i}\left[z\left(x_{i}\right)-z_{K E D}^{*}\left(x_{0}\right)\right]^{2} .
$$

Por meio da validação cruzada, pode-se calcular o erro verdadeiro em todos os pontos amostrais, assim como a variância de interpolação. A partir destas medidas de incerteza pode-se derivar o número de desvios padrão de interpolação (Yamamoto, 2005), como:

$$
n_{s_{0}}\left(x_{0}\right)=-\left[z_{K E D}^{*}\left(x_{0}\right)-z\left(x_{0}\right)\right] \text { para } s_{0} \neq 0 .
$$

Realizada a validação cruzada, a variável número de desvios padrão de interpolação é conhecida em cada ponto amostral. Esta nova variável é interpolada em cada nó da malha regular a ser estimada. Ao se estimar a variável de interesse através da krigagem com deriva externa, pode-se obter também o desvio padrão de interpolação associado à estimativa $z_{K E D}^{*}\left(x_{o}\right)$. Assim, obtém-se para cada nó da malha regular a estimativa $z_{K E D}^{*}\left(x_{o}\right)$, o número de desvios padrão de interpolação $n_{S_{0}}\left(x_{0}\right)$ e o desvio padrão de interpolação $s_{0}$. Multiplicando-se estas duas medidas de incerteza deriva-se a "quantidade de correção" que será somada à estimativa, conforme:

$$
z_{K E D}^{* *}\left(x_{0}\right)=z_{K E D}^{*}\left(x_{0}\right)+n_{s_{0}}\left(x_{0}\right) s_{0}
$$

onde $z_{K E D}^{* *}\left(x_{0}\right)$ é o valor corrigido da krigagem com deriva externa; $n_{S_{0}}\left(x_{0}\right) s_{0}$ é a "quantidade de correção" aplicada. Maiores detalhes sobre a correção do efeito de suavização podem ser obtidos em Yamamoto (2005) e em Yamamoto (2007).

\section{GEOLOGIA DA ÁREA}

A área de pesquisa localiza-se na porção NE do município de Fortaleza no estado do Ceará (Figura 1). Na região 
predominam sedimentos tercio-quaternários da Formação Barreiras e sedimentos arenosos, pleistocênicos a quaternários, de paleodunas, dunas e praias. Geomorfologicamente, os primeiros sedimentos são constituintes dos Tabuleiros Pré-litorâneos e os segundos formam a Planície Litorânea.

Cavalcanti et al. (1998) descrevem nestes domínios geomorfológicos três unidades geológicas principais, a saber:

1. Formação Barreiras: composta por sedimentos areno-argilosos, consolidados a inconsolidados, mal selecionados, com granulação variando de fina a média e com níveis conglomeráticos e lateríticos.

2. Aluviões: compostos por areias mal selecionadas com granulação variando de média a grossa. Nas planícies de inundação, os sedimentos são essencialmente silto-argilosos com alguma areia e ricos em matéria orgânica.

3. Dunas: constituída por grandes depósitos eólicos compostos por areias esbranquiçadas, bem selecionadas, de granulação fina a média, quartzosas, com grãos foscos e arredondados.

\section{ANÁLISE ESTATÍSTICA}

Neste trabalho, a variável primária, cota potenciométrica, constitui uma amostra com 110 unidades amostrais, enquanto a variável secundária, cota altimétrica, está presente nos 110 pontos amostrais da variável primária e em outros 975 coincidentes com os centros das células que serão co-estimados. Na Figura 2, observam-se os pontos de dados da variável primária e, na Figura 3 A e B, os pontos de dados da cota altimétrica nos mesmos pontos da cota potenciométrica e em cada centro de célula com as informações da variável secundária. Destaca-se que os pontos possuem diâmetro proporcional ao valor das variáveis.

A Tabela 1 mostra os resultados obtidos para as estatísticas descritivas das três variáveis estudadas.

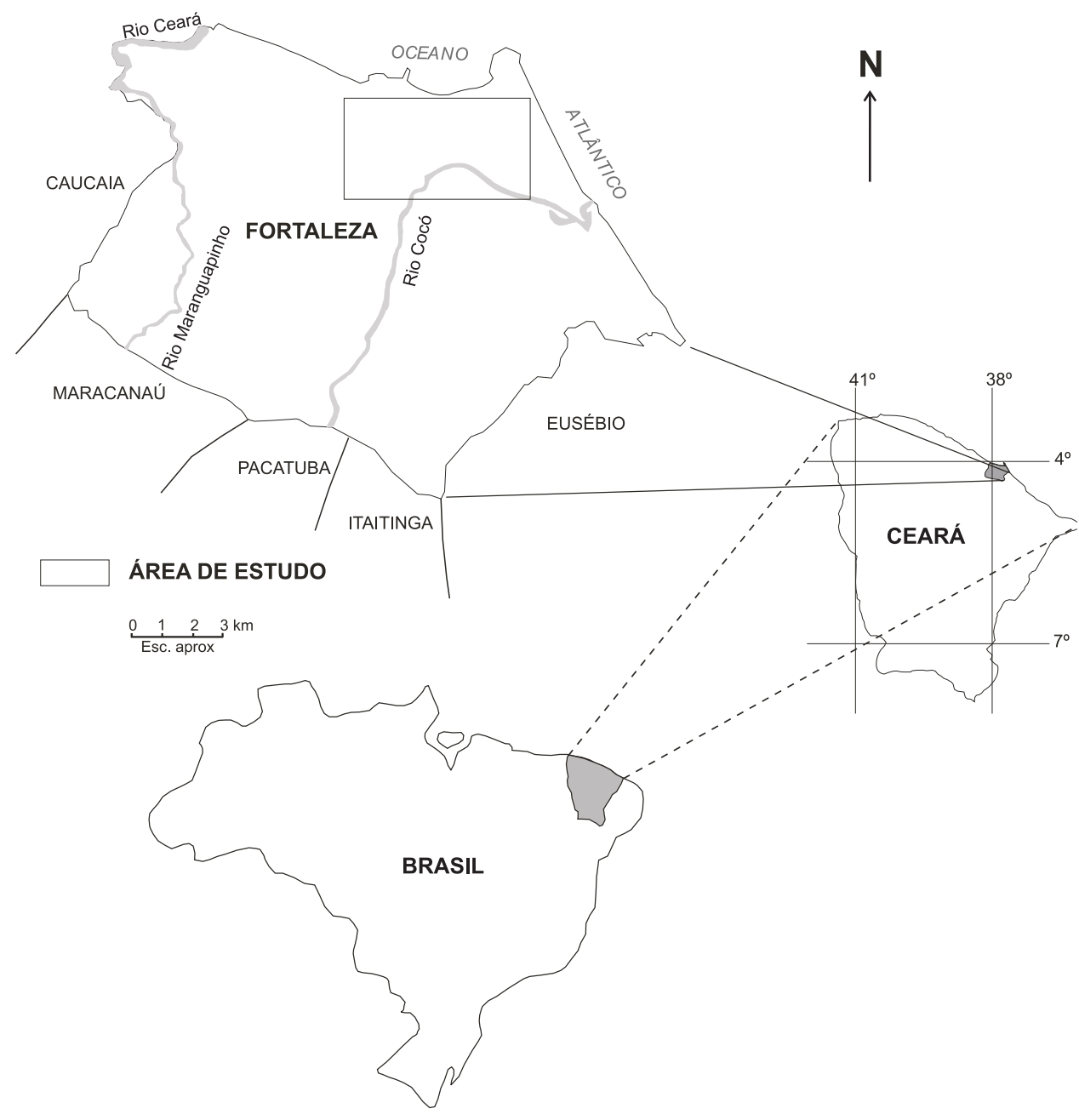

Figura 1. Mapa de localização da área de estudo. 

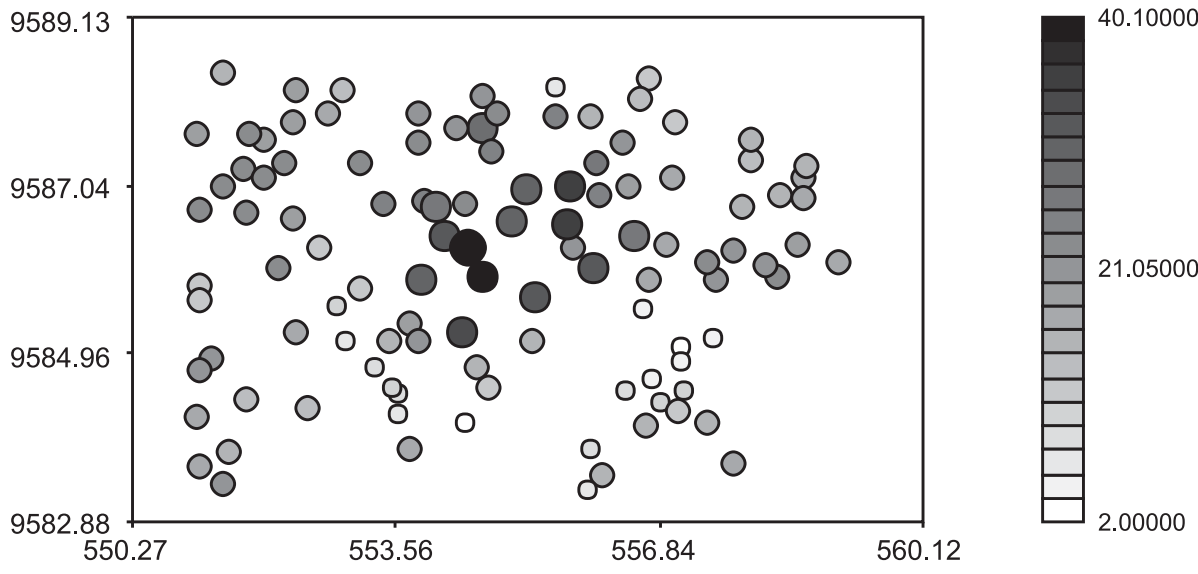

Figura 2. Mapa base de vp.
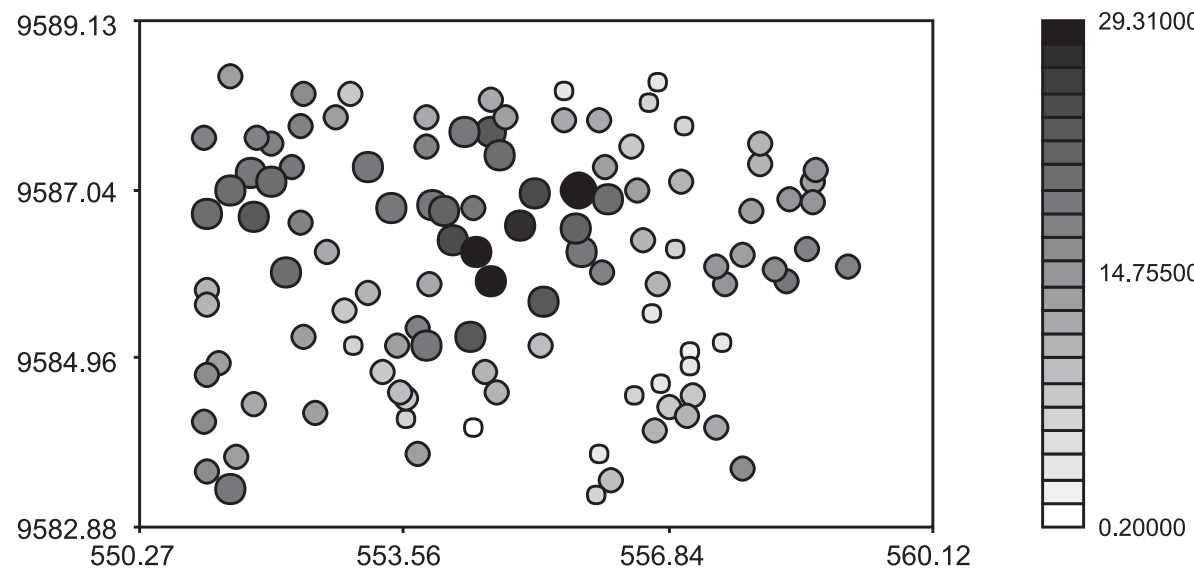

Figura 3A. Mapa base de vs na mesma posição que vp.
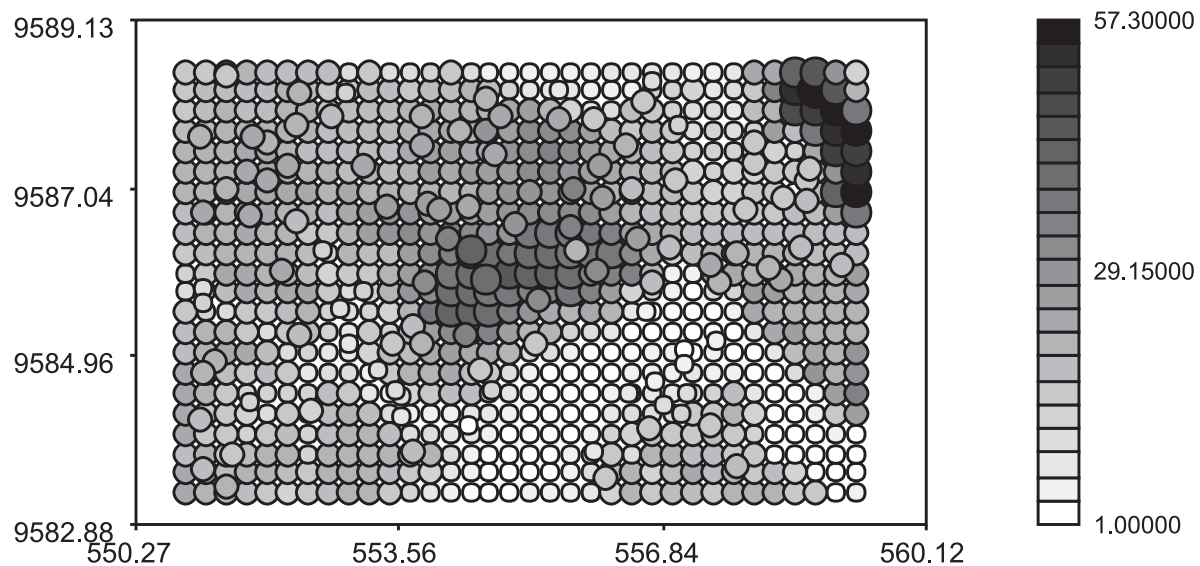

Figura 3B. Mapa base vs colocalizada. 
Tabela 1. Estatísticas descritivas das variáveis cota potenciométrica, cota altimétrica e cota altimétrica colocalizada.

\begin{tabular}{cccccc}
\hline $\begin{array}{c}\text { Variável } \\
\text { estatística }\end{array}$ & $\mathbf{n}$ & $\bar{x}$ & $\mathbf{s}$ & $\mathbf{C V}$ & Mediana \\
\hline CP & 110 & 13,304 & 6,133 & 0,461 & 13 \\
CA & 110 & 17,516 & 7,746 & 0,442 & 17 \\
CAC & 975 & 16,807 & 11,189 & 0,666 & 16,86 \\
\hline
\end{tabular}

Como se pode observar no histograma da Figura 4, a variável cota potenciométrica apresenta pequena assimetria positiva, tendendo a uma distribuição simétrica e baixa dispersão de valores. A variável cota altimétrica também é assimétrica positiva, porém com assimetria mais acentuada do que a anterior, como pode ser observado na Figura 5. Dos conjuntos de dados avaliados, aquele que contém a variável cota altimétrica em todos os nós das células a serem estimadas é o que apresenta assimetria positiva mais pronunciada e, consequentemente, maior grau de dispersão, o histograma dessa variável pode ser observado na Figura 6.

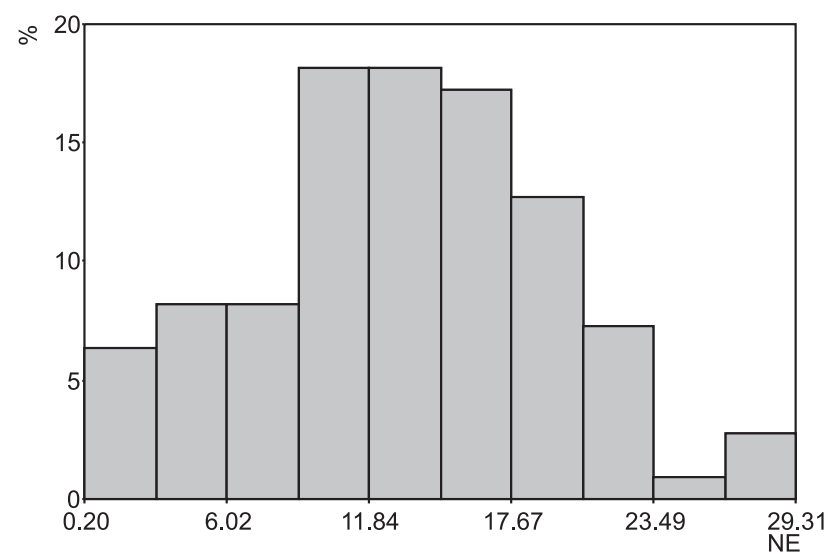

Figura 4. Histograma da variável cota potenciométrica.

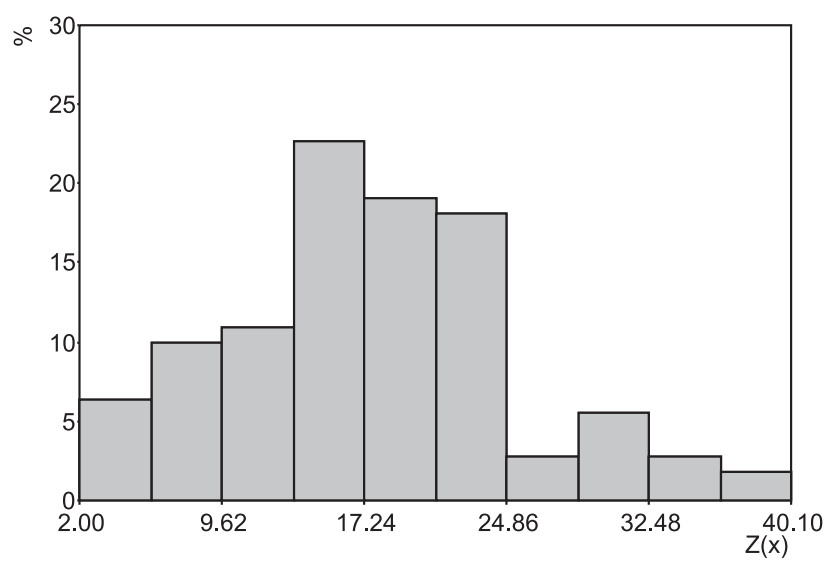

Figura 5. Histograma da variável cota altimétrica.

\section{ANÁLISE ESTATÍSTICA BIVARIADA}

A existência de correlação espacial entre variáveis é um pré-requisito básico para o cálculo da cokrigagem e também para a estimativa pelo método da krigagem com deriva externa.

A Figura 7 ilustra o diagrama de dispersão entre as variáveis cota potenciométrica e cota altimétrica. Pode-se observar que a nuvem de pontos apresenta pequena dispersão em torno da reta de regressão, indicando alta correlação entre as variáveis, o que também é verificado pelo coeficiente de correlação, igual a 0,88, e pela reta de regressão que é: $y=1,095+0,697 x$.

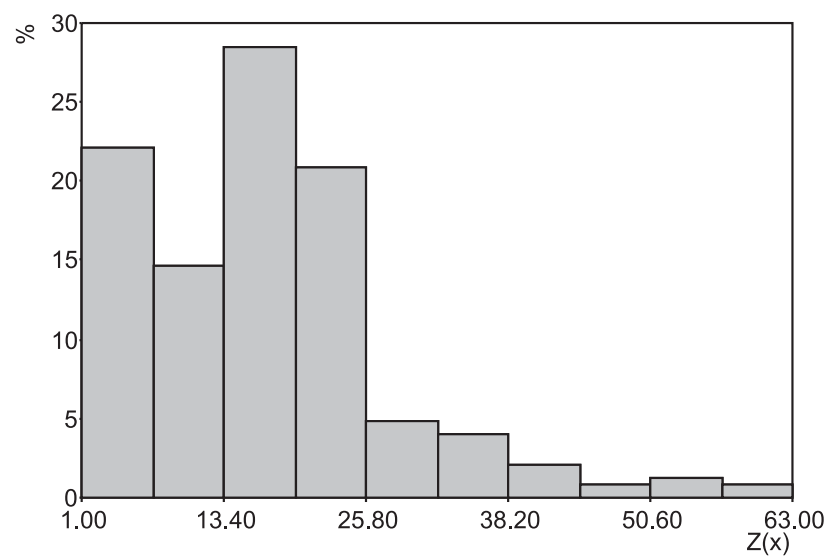

Figura 6. Histograma da variável cota altimétrica colocalizada.

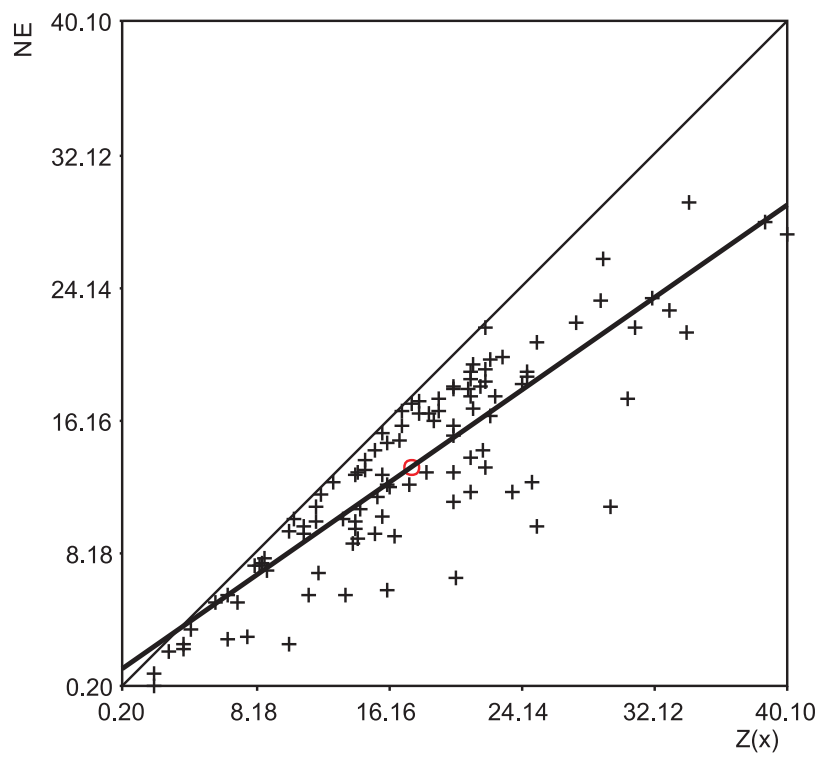

Figura 7. Diagrama de dispersão entre a variável cota potenciométrica e cota altimétrica. 


\section{ANÁLISE GEOESTATÍSTICA}

Feita a análise exploratória dos dados definiu-se a existência de anisotropia com direção de maior continuidade $90^{\circ}$ e a de menor continuidade a de $0^{\circ}$ para todas as variáveis, como podem ser observadas nas Figuras 8 a 10, que são, respectivamente, os dois variogramas diretos para as variáveis cota potenciométrica e cota altimétrica e, por fim, o variograma cruzado de ambas variáveis, todos com os modelos teóricos ajustados. Os parâmetros de ajuste podem ser observados na Tabela 2

Devem ser feitas duas observações, a primeira diz respeito ao variograma da variável cota altimétrica que levou em consideração a base de dados completa, ou seja, a base de dados com heterotopia parcial, o que confere ao gráfico o aspecto suavizado que pode ser observado na Figura 9. A segunda observação diz respeito ao modelo linear de corregionalização que é respeitado, uma vez que as condições de positividade definidas foram atendidas.

$$
+=0 \quad \diamond=90
$$



Figura 8. Variograma experimental e respectivo modelo ajustado para a variável cota potenciométrica.

$$
+=0 \quad \diamond=90
$$

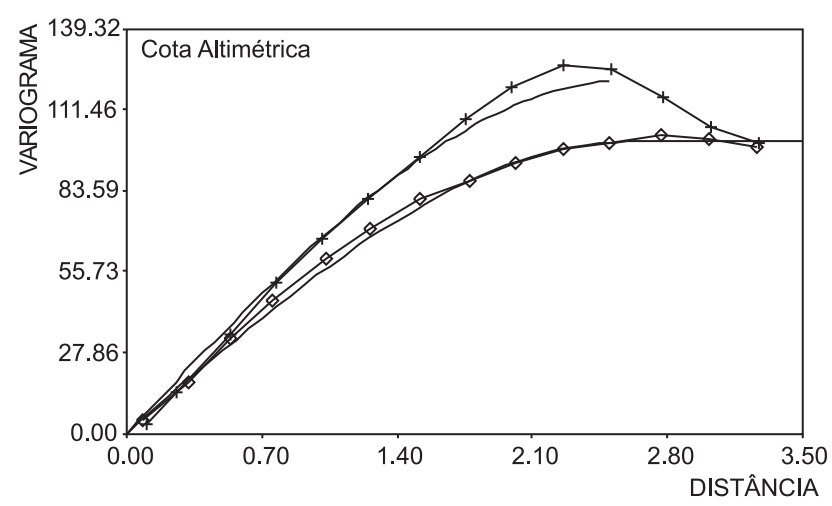

Figura 9. Variograma experimental e respectivo modelo ajustado para a variável cota altimétrica.

$$
+=0 \quad \diamond=90
$$

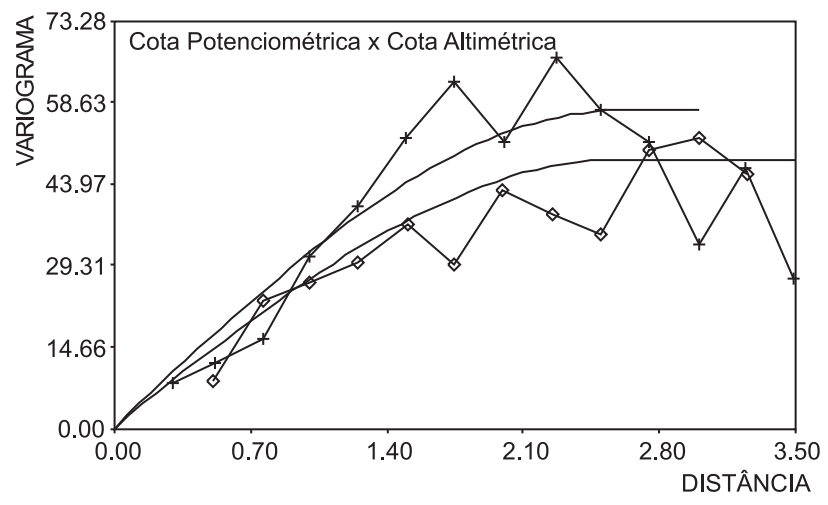

Figura 10. Variograma experimental cruzado entre as variáveis cota potenciométrica e cota altimétrica e respectivo modelo ajustado.

Tabela 2. Parâmetros do modelo teórico de variograma ajustado ao variograma experimental da variável cota potenciométrica $(C P)$; cota altimétrica $(C A)$ e ao variograma cruzado entre a cota potenciométrica e a cota altimétrica.

\begin{tabular}{ccccccc}
\hline Variável & Estrutura & Modelo & $\mathbf{C}_{0}$ & $\mathbf{C}$ & $\mathbf{A}_{\max }$ & $\mathbf{A}_{\min }$ \\
\hline \multirow{2}{*}{$\mathrm{CP}$} & 1 & Esférico & \multirow{2}{*}{0,6} & 38 & 2,6 & 2,6 \\
& 2 & Esférico & & 9,1 & $\infty$ & 2,6 \\
$\mathrm{CA}$ & 1 & Esférico & 0 & 99,8 & 2,6 & 2,6 \\
& 2 & Esférico & 0,6 & 21,17 & $\infty$ & 2,6 \\
$\mathrm{CP} \times \mathrm{CA}$ & 1 & Esférico & & 47,8 & 2,6 & 2,6 \\
& 2 & Esférico & 0,6 & 8,8 & $\infty$ & 2,6 \\
\hline
\end{tabular}

Conforme já afirmado, para a krigagem com deriva externa, o variograma calculado deve ser o dos resíduos entre a variável primária e a secundária. Este variograma pode ser observado na Figura 11 que é o variograma experimental calculado para quatro direções. Neste gráfico, a direção de $0^{\circ}$ apresenta ausência de estrutura, já as outras três direções apresentam variações pouco significativas. Deste modo, optou-se pelo modelo de variância espacial isotrópico e o variograma calculado para posterior ajuste foi o omnidirecional, conforme pode ser observado na Figura 12. Na Tabela 3 apresentam-se os parâmetros do ajuste realizado.

Tabela 3. Parâmetros do modelo teórico de variograma ajustado ao variograma experimental dos resíduos entre a cota potenciométrica (CP) e a cota altimétrica (CA).

\begin{tabular}{ccccccc}
\hline Variável & Estrutura & Modelo & $\mathbf{C}_{\mathbf{0}}$ & $\mathbf{C}$ & $\mathbf{A}_{\max }$ & $\mathbf{A}_{\min }$ \\
\hline Resíduo & 1 & Esférico & 0 & 4,58 & 1,1 & 1,1 \\
CP x CA & 2 & Esférico & & 4,032 & 1,7 & 1,7 \\
\hline
\end{tabular}




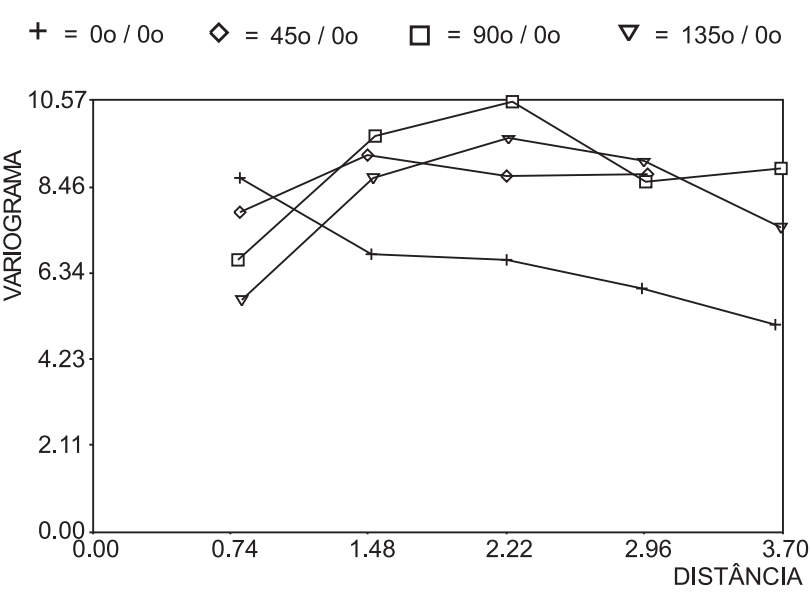

Figura 11. Variograma dos resíduos calculado em quatro direções.

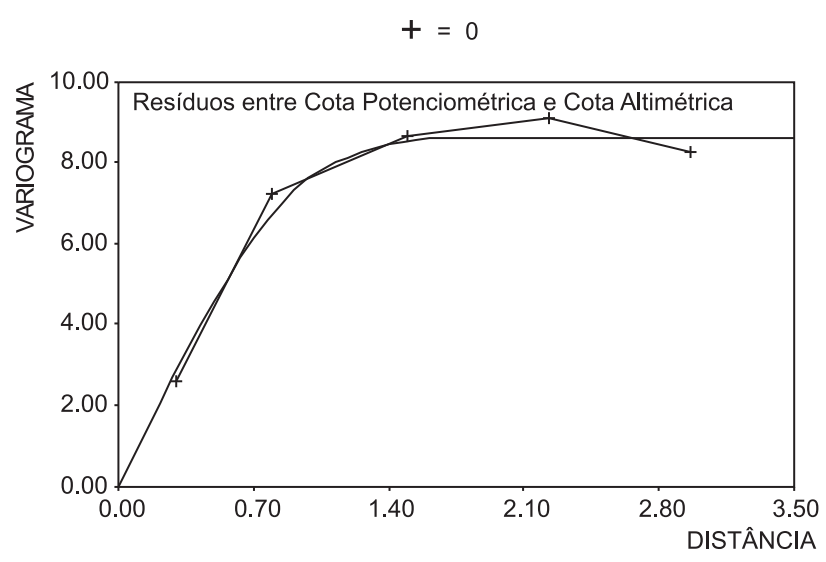

Figura 12. Variograma e modelo teórico omnidirecional.

\section{ESTIMATIVA POR COKRIGAGEM ORDINÁRIA}

A estimativa por cokrigagem ordinária foi calculada para a variável cota potenciométrica, como informação primária, e a cota altimétrica, amostrada em heterotopia parcial em relação à primeira variável, como informação secundária. Os cálculos realizados utilizaram o modelo de blocos contendo 32 blocos ao longo da direção Leste e 21 na direção Norte, totalizando 672 blocos com abertura de $0,25 \mathrm{~km}$ em ambas as direções.

Optou-se por utilizar parâmetros de vizinhança pouco restritivos, como pode ser observado na Tabela 4, dado o caráter heterotópico dos dados. A Figura 13 apresenta o mapa de estimativas da cota potenciométrica.

Pode-se observar na Figura 13 que a estimativa é suavizada, o que é confirmado pelas informações de máximo e mínimo da estimativa, as quais são maiores e menores, respectivamente, do que os valores de mínimo e máximo amostrais.

\section{ESTIMATIVA POR KRIGAGEM COM DERIVA EXTERNA SEM CORREÇÃO DO EFEITO DE SUAVIZAÇÃO}

Assim como para a cokrigagem ordinária, este método foi utilizado para estimar a cota potenciométrica, considerando as informações da variável cota altimétrica. Os parâmetros de vizinhança são aqueles definidos no método de estimativa anterior, conforme apresentado na Tabela 4.

Com a finalidade de comparar os resultados dos vários métodos e também devido à exigência da KDE de colocalização dos dados, o modelo de blocos utilizado possui as mesmas características daquele definido para a cokrigagem ordinária. O mapa de estimativas por krigagem com deriva externa é o apresentado na Figura 14.

Os resultados obtidos por este método apresentam-se menos suavizados que os do método discutido anteriormente. Deve-se observar que, embora a amplitude dos valores de resultados seja maior do que a obtida pela cokrigagem ordinária, esta ainda não atinge os mesmos valores da amostra. Os valores de máximo e mínimo da amostra não são alcançados.

\section{ESTIMATIVA POR KRIGAGEM COM DERIVA EXTERNA COM CORREÇÃO DO EFEITO DE SUAVIZAÇÃO}

A krigagem com deriva externa com correção do efeito de suavização foi realizada utilizando rigorosamente os mesmos parâmetros definidos para a cokrigagem ordinária e a krigagem com deriva externa sem a aplicação do algoritmo de correção do efeito de suavização.

A Figura 15 apresenta as estimativas corrigidas. Pela análise desta figura a correção minimizou a suavização e ainda aproximou os valores de máximo e mínimo estimados, daqueles amostrais. Observa-se ainda que a suavização obtida pela cokrigagem ordinária (Figura 13) é maior se comparada aos resultados deste método, sobretudo na porção centro-norte da área.

Comparando os resultados dos três algoritmos com a variável secundária, observa-se que o pior desempenho foi obtido pela cokrigagem, pois é este método que apresenta o menor coeficiente de correlação, como pode ser observado na Figura 16. Os resultados da krigagem com deriva externa com correção do efeito de suavização foram inferiores àqueles sem correção, devido à propagação dos erros (número de desvios padrão de interpolação) obtidos na fase da validação cruzada. Observando-se o mapa base da variável primária (Figura 3), pode-se verificar a existência de buracos na amostragem, os quais são responsáveis pela baixa qualidade das estimativas. Portanto, este método deve ser aplicado em situações em que a amostragem esteja com melhor distribuição espacial, ou seja, uniformemente distribuídas na região de estudo. 
Tabela 4. Parâmetros de vizinhança utilizados nas estimativas.

\begin{tabular}{ccccc}
\hline Variável & $\begin{array}{c}\text { Setores } \\
\text { Angulares }\end{array}$ & $\begin{array}{c}\mathbf{N}^{\circ} \text { ótimo } \\
\text { de } \\
\text { pontos }\end{array}$ & $\begin{array}{c}\mathbf{N}^{\circ} \text { min. } \\
\text { de } \\
\text { pontos }\end{array}$ & Distância \\
\hline $\begin{array}{c}\text { Cota } \\
\text { potenciométrica } \\
\begin{array}{c}\text { Cota } \\
\text { altimétrica }\end{array}\end{array}$ & 4 & 1 & 1 & 8,85 \\
\hline
\end{tabular}

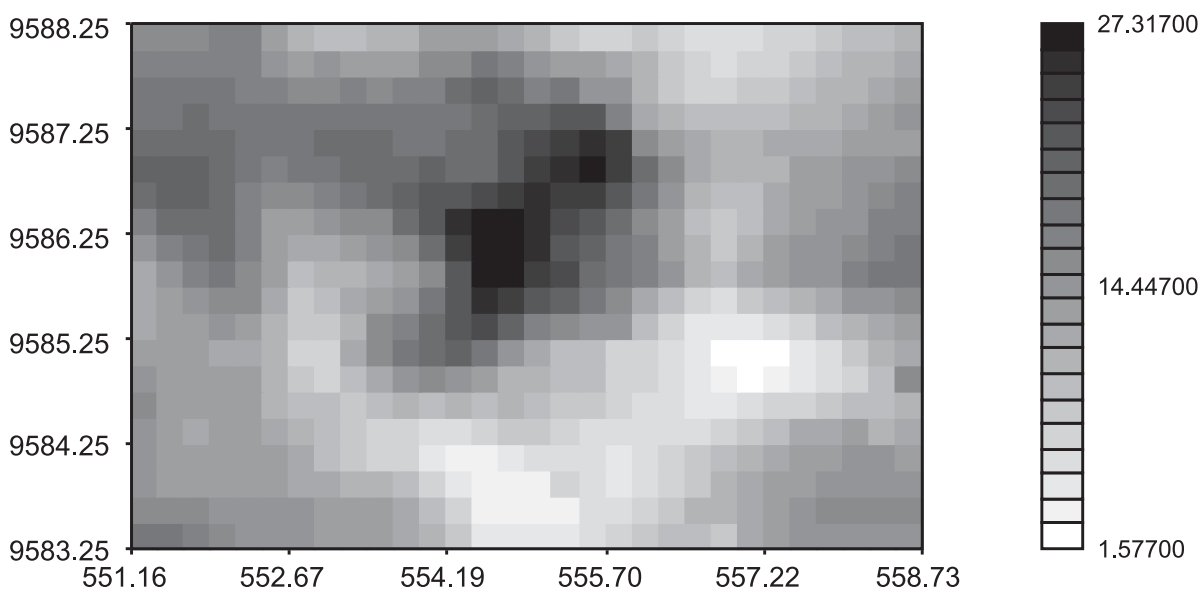

Figura 13. Mapa da cokrigagem ordinária para a variável cota potenciométrica.
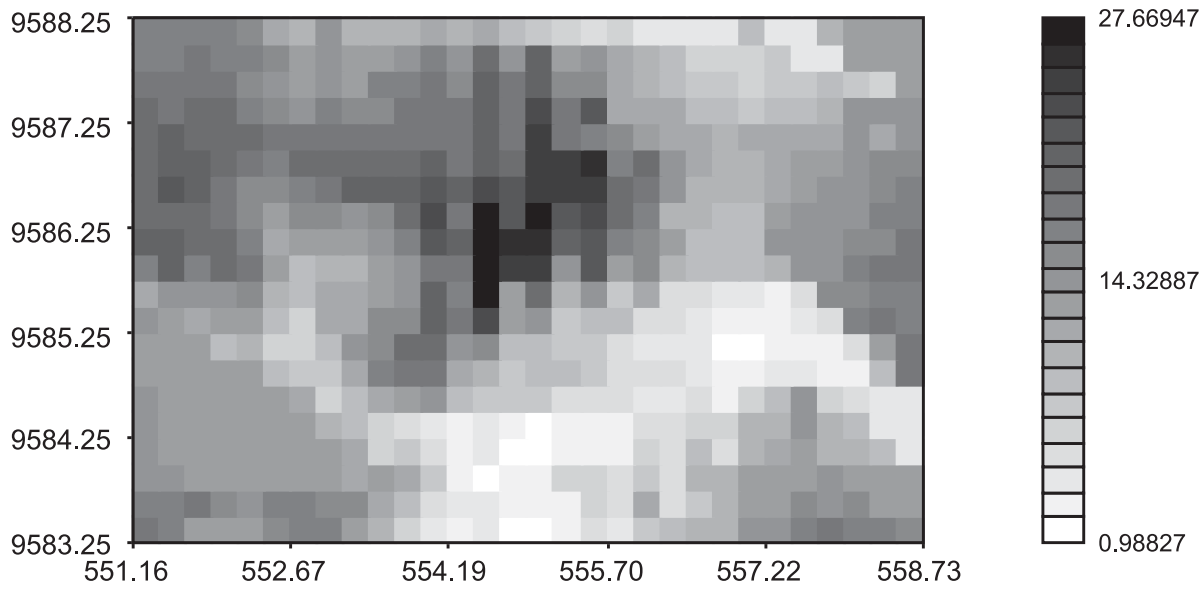

Figura 14. Mapa da krigagem com deriva externa para a variável cota potenciométrica. 


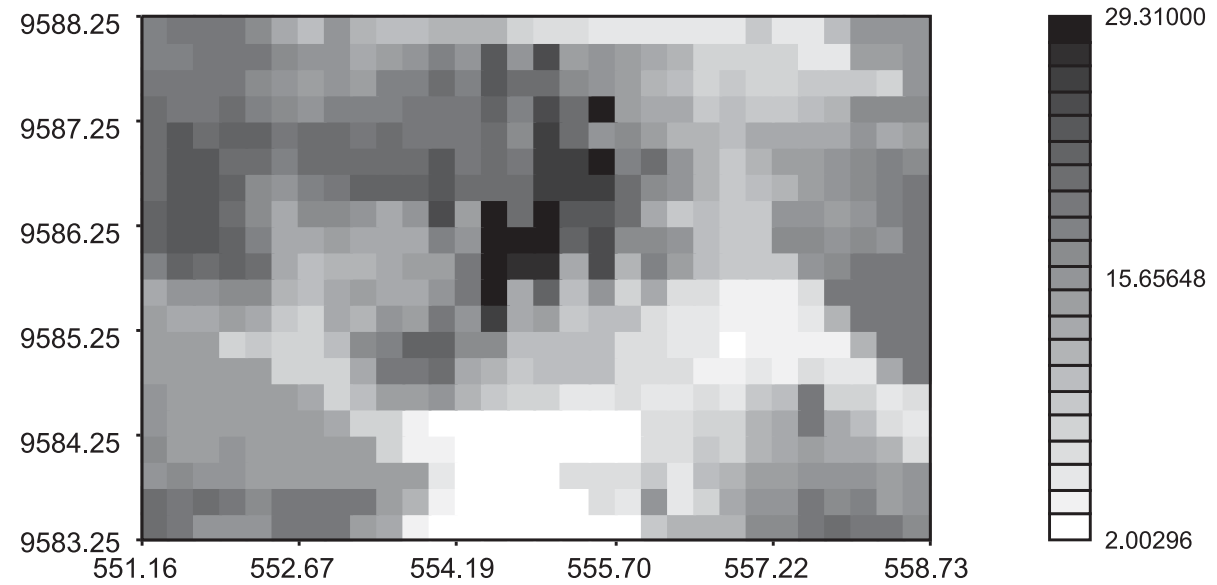

Figura 15. Mapa da krigagem com deriva externa, com correção do efeito de suavização, para a variável cota potenciométrica.

A

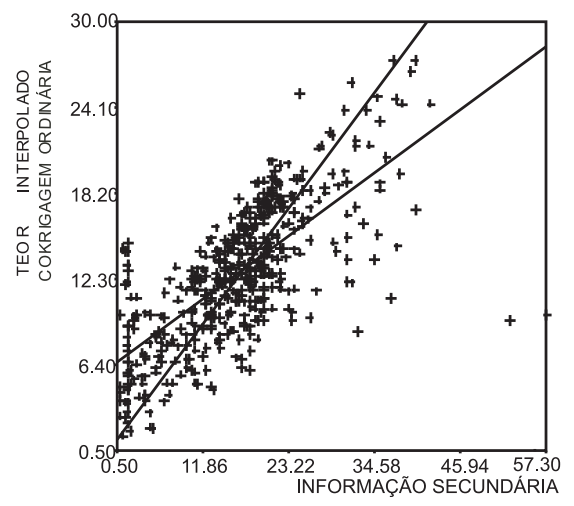

B

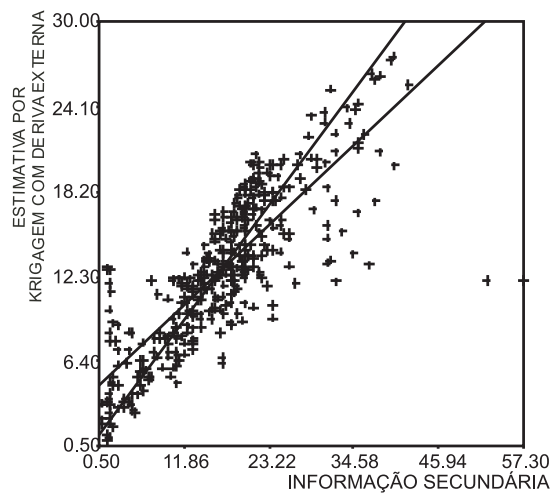

C

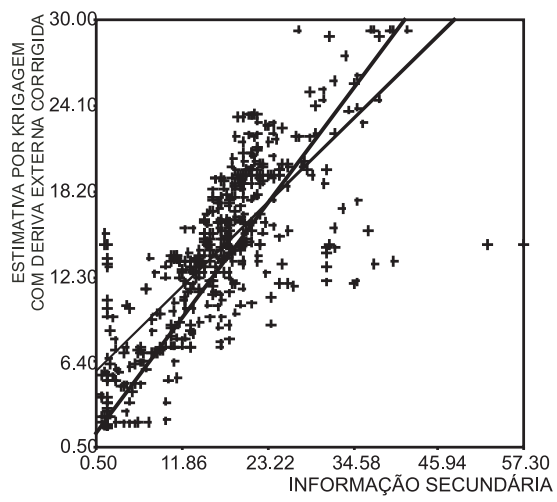

Figura 16. Diagramas de dispersão entre a cota altimétrica e a cota potenciométrica estimada por A. cokrigagem ordinária; B. krigagem com deriva externa e C. krigagem com deriva externa com correção do efeito de suavização.

\section{CONCLUSÕES}

A comparação entre estes três métodos de estimativa multivariados visou verificar qual a resposta mais adequada ao estudo de caso em questão. Esta verificação levou em consideração a análise dos mapas estimados, a análise dos diagramas de dispersão entre a cota altimétrica e a potenciométrica da amostra, comparados com as estimativas e as informações de cota altimétrica colocalizadas.

A cokrigagem ordinária é o método mais trabalhoso, devido ao grande número de variogramas considerado. A grande dificuldade está na verificação da condição de positividade exigida pelo modelo linear de corregionaliza- ção. O sistema de equações de cokrigagem ordinária nem sempre é estável devido às condições de restrições a serem satisfeitas.

A krigagem com deriva externa apresenta a vantagem de permitir a correção do efeito de suavização. Adicionalmente, este método necessita apenas do cálculo e modelagem de um único variograma experimental. O sistema de equações de krigagem com deriva externa é sempre estável, mas a qualidade das estimativas, ou seja, a precisão local depende do coeficiente de correlação entre as variáveis primária e secundária. Nesse caso, a correlação amostral foi igual a 0,88 e, portanto, os resultados da krigagem com deriva externa foram superiores em relação à cokrigagem ordinária. 


\section{REFERÊNCIAS BIBLIOGRÁFICAS}

CAVALCANTI, V. M. M.; SENA, R. B.; RIBEIRO, J. A. P.; COLARES, J. Q. S; GOMES, F. E. M. Plano diretor de mineração para a região metropolitana de Fortaleza. Brasília: DNPM/CPRM, 1998. 191 p. (Série Difusão Tecnológica, 7).

FONETELES, H. R. da N.; YAMAMOTO, J. K.; ROCHA, M. M.; GANDOLFI, N. Geoestatística aplicada à modelagem da superfície freática a partir de dados do nível estático: o caso da porção nordeste de Fortaleza (CE). Solos e rochas. Revista Brasileira de Geotecnia, v. 29, n. 3, p. 331-339, 2006.

ISAAKS, E. H.; SRIVASTAVA, R. M. An introduction to applied geostatistics. New York: Oxford University Press, 1989. $561 \mathrm{p}$.

JOURNEL, A. G.; KYRIAKIDIS, P. C.; MAO S. Correcting smoothing effect of estimators: a spectral postprocessor. Mathematical Geology, v. 32, n. 7, p. 787-813, 2000.

OLEA, R.; PAWLOWSKY, V. Compensating for estimation smoothing in kriging. Mathematical Geology, v. 28, n. 4, p. 407-417, 1996.

STURARO, J. R.; LANDIM, P. M. Técnica geoestatística para extrapolação da superfície potenciométrica em centros urbanos. CONGRESSO BRASILEIRO DE GEOLOGIA, 38., 1994, Camboriu. Anais... Camboriu: SBG, 1994. p. 511-512. v. 1.

WACKERNAGEL, H. Multivariate geostatistics. Berlin: Springer-Verlag, 1998. 291 p.

YAMAMOTO, J. K. A Review of numerical methods for the interpolation of geological data. Anais da Academia Brasileira de Ciências, v. 70, n. 1, p. 91-116, 1998.

YAMAMOTO, J. K. An alternative measure of the reliability of ordinary kriging estimates. Mathematical Geology, v. 32, n. 4, p. 489-509, 2000.

YAMAMOTO, J. K. Correcting the smoothing effect of ordinary kriging estimates. Mathemathical Geology, v. 32, p. 69-94, 2005.

YAMAMOTO, J. K. On unbiased backtransform of lognormal kriging estimates. Computational Geosciences, v. 11, n. 3, p. 219-234, 2007. 